\title{
Co-optimization of Generation Unit Commitment and Transmission Switching with N-1 Reliability
}

\author{
Kory W. Hedman, Student Member, IEEE, Michael C. Ferris, Richard P. O’Neill, Emily B. Fisher, Student \\ Member, IEEE, and Shmuel S. Oren, Fellow, IEEE
}

\begin{abstract}
Currently, there is a national push for a smarter electric grid, one that is more controllable and flexible. The full control of transmission assets are not currently built into electric network optimization models. Optimal transmission switching is a straightforward way to leverage grid controllability: to make better use of the existing system and meet growing demand with existing infrastructure. Previous papers have shown that optimizing the network topology improves the dispatch of electrical networks. Such optimal topology dispatch can be categorized as a smart grid application where there is a cooptimization of both generators and transmission topology. In this paper we present a co-optimization formulation of the generation unit commitment and transmission switching problem while ensuring $\mathrm{N}-1$ reliability. We show that the optimal topology of the network can vary from hour to hour. We also show that optimizing the topology can change the optimal unit commitment schedule. This problem is large and computationally complex even for medium sized systems. We present decomposition and computational approaches to solving this problem. Results are presented for the IEEE RTS 96 test case.
\end{abstract}

Index Terms-Generation unit commitment, integer programming, power generation dispatch, power system economics, power system reliability, power transmission control, power transmission economics

\begin{tabular}{ll}
\multicolumn{2}{c}{ NomENCLATURE } \\
\multicolumn{1}{c}{ Indices } & \multicolumn{1}{c}{ Operating state; $c=0$ indicates the no contingency } \\
& state (steady-state); $c>0$ is a single contingency state \\
$g:$ & Generator \\
$g(n):$ & Set of generators at node $n$ \\
$k:$ & Transmission element (line or transformer) \\
$k(n,):$. & Set of transmission assets with $n$ as the 'to' node \\
$k(., n):$ & Set of transmission assets with $n$ as the 'from' node \\
$m, n:$ & Nodes \\
$t:$ & Time period
\end{tabular}

\footnotetext{
Parameters

$B_{k}$ : $\quad$ Electrical susceptance of transmission element $k$

$c_{g}$ : $\quad$ Production cost for generator $g$

$c_{g}^{S U}: \quad$ Startup cost for generator $g$
}

Manuscript received January 15, 2009; revised June 1, 2009 and September 26, 2009.

Kory W. Hedman and Shmuel S. Oren are with the Department of Industrial Engineering and Operations Research, University of California at Berkeley, Berkeley, CA 94720-1777 USA (email: kwh@ieor.berkeley.edu; oren@ieor.berkeley.edu).

Michael C. Ferris is with the Department of Computer Sciences, University of Wisconsin at Madison, Madison, WI 53706 USA (email: ferris@cs.wisc.edu).

Richard P. O'Neill is with the Federal Energy Regulatory Commission (FERC), Washington, DC 20426 USA (email: richard.oneill@ferc.gov).

Emily B. Fisher is with the Johns Hopkins University, Baltimore, MD 21218 USA (email: ebartho3@jhu.edu).

\begin{tabular}{|c|c|}
\hline$c^{S D}{ }_{g}$ & Shutdown cost for generator $g$ \\
\hline$d_{n t}:$ & Real power load at node $n$ for period $t$ \\
\hline$D T_{g}, U T_{g}:$ & Minimum down time and min up time for generator $g$ \\
\hline$M_{k}:$ & Big M value for transmission element $k$ \\
\hline$N 1_{e c}:$ & $\begin{array}{l}\text { Binary parameter that is } 0 \text { when the element } e \text { is the } \\
\text { contingency and } c>0,1 \text { otherwise }\end{array}$ \\
\hline$P_{g}^{\max }, P_{g}^{\min }$ & Max and min capacity of generator $g$ \\
\hline$P_{k c}^{\max }, P_{k c}^{\min }:$ & Max and min rating of transmission element $k$, state $c$ \\
\hline$R_{g}^{+}:$ & Maximum ramp up rate for generator $g$ \\
\hline$R_{g}^{-}:$ & Maximum ramp down rate for generator $g$ \\
\hline$R^{S D}:$ & Maximum shutdown ramp rate for generator $g$ \\
\hline$R_{g}^{S U_{g}^{\circ}}$ & Maximum startup ramp rate for generator $g$ \\
\hline & Number of periods \\
\hline$\theta^{\max }, \theta^{\min }:$ & Max and min bus voltage angle \\
\hline \multicolumn{2}{|l|}{ Variables } \\
\hline$P_{g c t}$ & $\begin{array}{l}\text { Real power supply from generator } g \text { at node } n \text { for state } \\
c \text { and period } t\end{array}$ \\
\hline$P_{k c t}:$ & $\begin{array}{l}\text { Real power flow from node } m \text { to } n \text { for transmission } \\
\text { element } k \text {, state } c \text {, and period } t\end{array}$ \\
\hline$u_{g t}$ : & $\begin{array}{l}\text { Binary unit commitment variable for generator } g \text { anc } \\
\text { period } t(0 \text { offline/down, } 1 \text { online/operational })\end{array}$ \\
\hline$v_{g t}$ : & $\begin{array}{l}\text { Startup variable for generator } g \text { and period } t \text { ( } 1 \text { for } \\
\text { startup, } 0 \text { otherwise) }\end{array}$ \\
\hline$w_{g t}:$ & $\begin{array}{l}\text { Shutdown variable for generator } g \text { and period } t \text { ( } 1 \text { for } \\
\text { shutdown, } 0 \text { otherwise) }\end{array}$ \\
\hline$z_{k t}:$ & $\begin{array}{l}\text { Binary variable for transmission element } k \text { and perioc } \\
t(0 \text { open/not in service, } 1 \text { closed/in service })\end{array}$ \\
\hline$\theta_{n c t}:$ & Voltage angle at node $n$ for state $c$ and period $t$ \\
\hline
\end{tabular}

\section{INTRODUCTION}

THE physics that govern electrical networks make them unique. It is possible to remove a link and improve the efficiency of the system. Traditionally, transmission networks for bulk power flow have been modeled as static, except during times of forced outages or maintenance. This traditional view does not describe them as assets that operators have the ability to control. However, switching transmission lines is a common practice with a mature technology; circuit breakers can open and close transmission lines.

System operators can and do change the topology of systems to improve system performance. Operators switch transmission elements to improve voltage profiles or increase transfer capacity [1]. For example, it is an accepted practice to open light-loaded transmission lines at night for better voltages profiles [2]. The Northeast Power Coordinating Council includes "switch out internal transmission lines" in the list of possible actions to avoid abnormal voltage conditions [3] [4]. In PJM, Special Protection Schemes (SPSs) allow the operator to disconnect a line during normal operations but return it to service during a contingency. These decisions are made under a set of prescribed rules by the operator, rather than included in the optimization formulation. 
Transmission switching has been explored as a control method for problems such as over or under voltage situations, line overloading [5]-[7], loss and/or cost reduction [8] [9], system security [10], or a combination of these [11]-[13]. Numerous SPSs address specific instances of switching during emergency conditions. Some SPSs open lines during emergency conditions, demonstrating that it can be beneficial to change the topology during emergency conditions. In this paper we present the concept of co-optimizing the network topology along with unit commitment.

Optimal transmission switching for economic benefit in a market context was investigated by O'Neill et al. [14]. Fisher et al. [15] and Hedman et al. [16] present and analyze the optimal transmission switching problem on the IEEE 118 bus test case and showed that co-optimizing the generation and the network topology can result in substantial savings. Transmission switching is not by definition incompatible with reliable network operations. Hedman et al. [17] demonstrated that transmission switching can be beneficial even while ensuring an $\mathrm{N}-1$ reliable network.

New transmission infrastructure can be expensive and hard to site [18]. Therefore, optimal use of the existing system and optimal expansion should be a priority. The US Energy Policy Act of 2005 includes a directive for federal agencies to "encourage...deployment of advanced transmission technologies," including "optimized transmission line configuration." This research is also in line with FERC Order 890 - to improve the economic operations of the electric transmission grid. It also addresses the items listed in Title 13 "Smart Grid" of the Energy Independence and Security Act of 2007: (1) "increased use of... controls technology to improve reliability, stability, and efficiency of the grid" and (2) "dynamic optimization of grid operations and resources."

Optimal transmission switching is a promising option because it uses existing hardware to achieve important and timely goals: increased grid flexibility and efficiency. In this paper, we examine the potential for switching to increase the economic efficiency of power system dispatch by cooptimizing transmission switching decisions and the system dispatch model. This creates an N-1 DCOPF multi-period generation unit commitment transmission switching model.

The paper is organized as follows. Section II discusses the model formulation, including a discussion on transmission switching, unit commitment, minimum up and down time constraints, etc. Section III discusses how the general model is modified for computational testing. A multi-period generation unit commitment optimal transmission switching model with $\mathrm{N}-1$ contingency constraints is difficult to solve and, therefore, we discuss in section III how we decompose and solve this optimization problem. Section IV presents a network overview and the results and analysis for the RTS 96 system. Section V discusses possible future work and Section VI concludes this paper.

\section{MOdEl Formulation}

This optimization problem is formulated as a Mixed Integer Programming (MIP) problem. The use of MIP within the electric industry is growing; PJM has switched from Lagrangian Relaxation (LR) to MIP for their generation unit commitment software [19] and for their real-time market lookahead [1]. These changes are estimated to save PJM over 150 million dollars per year [1] [19]. Furthermore, most US ISOs are testing and planning to switch to MIP in the near future [20].

\section{A. N-1 DCOPF Optimal Transmission Switching and Generation Unit Commitment Formulation}

The Direct Current Optimal Power Flow (DCOPF) is a commonly used linear approximation of the Alternating Current Optimal Power Flow (ACOPF). Fisher et al. [15] showed how the traditional DCOPF can incorporate transmission switching into the formulation. Within this paper, we are ensuring that the system is $\mathrm{N}-1$ compliant, i.e. the system can survive the loss of any single network component (generator or transmission element) in the system, except radial lines. This formulation also incorporates generation unit commitment in order to analyze how transmission switching affects unit commitment and vice versa.

The objective of the optimization problem is to minimize total cost, which includes the generator production costs in the no contingency case, the startup costs, and the shutdown costs (1). Since the demand is perfectly inelastic, minimizing the total cost is the same as maximizing the total social welfare. This objective is valid for systems where generation dispatch is a centralized process in which all operating costs are known. For systems where dispatch is determined by a centralized grid operator who takes bids, we optimize the bid surplus. In further discussion, we assume that bids are marginal costs.

The constraints represent the traditional power flow constraints that follow Kirchhoff's Laws, except for the modifications made to incorporate transmission switching. This is a lossless model, which allows us to use only one variable to represent a transmission element's power flow. Therefore, the node balance constraints, (3), account for flows to bus $n$ (injections) and flows from bus $m$ (withdrawals). If this were a lossy model, losses may increase or decrease, see [9], as a result of transmission switching. The objective is to minimize the total cost so even if losses increase, transmission switching can still be of value by decreasing the total cost. Constraints (4), (5a), and (5b) are modified to incorporate the decision to have a transmission element closed or open in the network. Injections into a bus are positive (generator supply, power flow to bus $n$ ) and withdrawals are negative (load, power flow from bus $m$ )

$$
\begin{gathered}
\text { Minimize: } \sum_{t} \sum_{g}\left(c_{g} P_{g 0 t}+c_{g}^{S U} v_{g t}+c_{g}^{S D} w_{g t}\right) \\
\text { s.t. } \\
\theta^{\min } \leq \theta_{n c t} \leq \theta^{\max }, \forall n, c, t \\
\sum_{\forall k(n, .)} P_{k c t}-\sum_{\forall k(., n)} P_{k c t}+\sum_{\forall g(n)} P_{g 0 t}=d_{n t}, \\
\forall n, c=0, \text { transmission contingency states } c, t \\
\sum_{\forall k(n, .)} P_{k c t}-\sum_{\forall k(., n)} P_{k c t}+\sum_{\forall g(n)} P_{g c t}=d_{n t}, \\
\forall n, \text { generator contingency states } c, t
\end{gathered}
$$

\footnotetext{
${ }^{1}$ See Sec.1223.a.5 of the US Energy Policy Act of 2005.
} 


$$
\begin{aligned}
& P_{k c}^{\min } N 1_{k c} z_{k t} \leq P_{k c t} \leq P_{k c}^{\max } N 1_{k c} z_{k t}, \forall k, c, t \\
& B_{k}\left(\theta_{n c t}-\theta_{m c t}\right)-P_{k c t}+\left(2-z_{k t}-N 1_{k c}\right) M_{k} \geq 0, \\
& \quad \forall k, c, t \\
& B_{k}\left(\theta_{n c t}-\theta_{m c t}\right)-P_{k c t}-\left(2-z_{k t}-N 1_{k c}\right) M_{k} \leq 0, \\
& \quad \forall k, c, t \\
& P_{g}^{\min } N 1_{g c} u_{g t} \leq P_{g c t} \leq P_{g}^{\max } N 1_{g c} u_{g t}, \forall g, c, t \\
& v_{g, t}-w_{g, t}=u_{g, t}-u_{g, t-1}, \forall g, t \\
& \quad \sum_{q=t-U T_{g}+1}^{t} v_{g, q} \leq u_{g, t}, \forall g, t \in\left\{U T_{g}, . ., T\right\} \\
& \quad \sum_{q=t-D T_{g}+1}^{t} w_{g, q} \leq 1-u_{g, t}, \forall g, t \in\left\{D T_{g}, . ., T\right\} \\
& P_{g 0 t}-P_{g 0, t-1} \leq R_{g}^{+} u_{g, t-1}+R_{g}^{S U} v_{g, t}, \forall g, t \\
& P_{g 0, t-1}-P_{g 0, t} \leq R_{g}^{-} u_{g, t}+R_{g}^{S D} w_{g, t}, \forall g, t \\
& P_{g c t}-P_{g 0, t} \leq R_{g}^{+}, \forall g, c, t \\
& P_{g 0, t} N 1_{g c}-P_{g c t} \leq R_{g}^{-}, \forall g, c, t \\
& 0 \leq v_{g, t} \leq 1, \forall g, t \\
& 0 \leq w_{g, t} \leq 1, \forall g, t \\
& u_{g, t} \in\{0,1\}, \forall g, t .
\end{aligned}
$$

\section{B. Contingency Modeling}

Each decision variable has a new value for each state $c$, except for $z_{k t}$ and the variables associated with the unit commitment formulation: $u_{g t}, v_{g t}$, and $w_{g t}$. State $c=0$ represents the no-contingency, steady-state variables and constraints whereas all other states represent single generator or (non radial) transmission contingencies.

We introduce a binary parameter for state $c$ and element $e$ : $N 1_{e c} . N 1_{k c}=0$ represents the loss of transmission element $k$; $N l_{g c}=0$ represents the loss of generator $g$. For $c=0, N 1_{e 0}=1$ for all $e$ as this state reflects steady-state operations. There are $N$ (transmission element or generator) contingencies. For $c>$ 0 ,

$$
\begin{aligned}
& N 1_{e c}=\left\{\begin{array}{c}
0, \text { if } c=e \\
1, \text { otherwise }
\end{array}\right\}, \forall c>0, e \\
& \sum_{\forall e} N 1_{e c}=N-1, \forall c>0 \\
& \forall \sum_{c>0} N 1_{e c}=N-1, \forall e .
\end{aligned}
$$

The binary parameter $N 1_{e c}$ forces the transmission element's flow to be zero within (4) when the transmission element is the contingency; the use of $N 1_{e c}$ in (6) forces a generator's supply to be zero when the generator is the contingency.

\section{Transmission Switching Modeling}

Constraints (5a) and (5b) ensure that if a transmission element is opened, these constraints are satisfied no matter what the values are for the corresponding bus angles. The transmission element is considered opened if it is the contingency, i.e. $N 1_{k c}=0$, or it is chosen to be opened as a result of transmission switching, i.e. $z_{k t}=0$.
In (5a) and (5b), $M_{k}$ is often called the "big M" value where $M_{k}$ is large enough to make the constraint nonbinding. $M_{k}$ must be a large number greater than or equal to $\mid B_{k}\left(\theta^{\max }\right.$ $\left.\theta^{\text {min }}\right)$. When either $z_{k t}=0$ or $N 1_{k c}=0, P_{k c t}$ is zero and the value of $M_{k}$ ensures that (5a) and (5b) are satisfied regardless of the difference in the bus angles. Without this adjustment to the power flow equations, the buses that were connected to this opened transmission element would be forced to have the same bus angle. With this adjustment, the solution corresponds to the case when the transmission element is open in the network, as desired.

ACOPF formulations include constraints on the angle difference between two connected buses; these constraints ensure angle stability. However, in a DCOPF model, an angle difference constraint would be redundant vis a vis the line flow capacity constraint, (4). Restricting the angle difference between connected buses is the same as placing a bound on the power flow on that line (in our formulation, $P_{k c t}$ in (5a) and (5b)). Thus, it is unnecessary to include both bus angle difference constraints and power flow constraints in a DCOPF formulation; instead, the power flow constraint can implicitly contain the limit on angle difference. If the physical limit on the angle difference is below a line's rated flow capacity, then the capacity limits can be adjusted to enforce the angle constraints. In the formulation presented here, we employ limits on each bus angle (2) since it is not redundant and it conveniently provides a lower bound on $M_{k}$.

The chosen min and max bus angle values are \pm 0.6 radians. It is computationally better for $M_{k}$ to be as small as possible, which would be $\left|B_{k}\left(\theta^{\max }-\theta^{\min }\right)\right|=1.2\left|B_{k}\right|$. A similar MIP optimization model (see [21]) is used for transmission expansion in which a shortest path problem is formulated to determine the minimum $M_{k}$ value. By using this technique, $M_{k}$ depends on the available paths between buses. Using this technique with a transmission switching model like ours would substantially increase solution difficulty. Transmission switching may remove previous paths between buses; thus, this technique would require $M_{k}$ to be a variable rather than a parameter. As a result, it is conducive to model the bus angle constraints by (2), making it possible to define $M_{k}$ as we previously stated.

All solutions from the N-1 DCOPF transmission switching problem must satisfy strict N-1 standards. For any contingency, the line capacity limits are defined by rate $\mathrm{C}$, or the emergency rating; otherwise, the capacity limits are equal to the steady-state, no contingency limits. We assume that when there is a transmission contingency, the generator dispatch levels do not change, which is the reason for the formulation presented in (3a). When there is a generator contingency, all of the on-line generators are allowed to adjust their output while satisfying (6) and ramping constraints (12) and (13) in order to survive the contingency. This associated re-dispatch cost is not included in the objective because the redispatch occurs in real time, whereas this model determines the short-term forward dispatch of the system. Since the probability of an outage is small, we are concerned with feasibility of surviving a contingency, not the cost. For further discussion on the N-1 DCOPF optimal transmission switching formulation, see [17]. 


\section{Generation Unit Commitment}

Generators are assumed to have linear generation costs, startup costs, and shutdown costs (1). We also assume that generators have minimum and maximum operating levels, see (6), minimum up and down time constraints, see (8) and (9), and ramp rate constraints, see (10)-(13).

The generator's state is represented by a unit commitment variable $u_{g t}$, which equals one (or zero) when the unit is on (or off). Offline generators cannot respond during a contingency. The startup binary variable is $v_{g t}$. When the unit is turned on in period $t, v_{g t}=1$; otherwise, $v_{g t}=0$. The shutdown binary variable, $w_{g t}$, equals one only when the unit is off in period $t$ and on for period $t-1$. By incorporating the startup and shutdown variables, we have the relationship in (7). This constraint can be found in papers that were published over forty years ago, [22]. Based on our chosen formulation, we are able to relax the integrality constraints of the startup and shutdown variables, which we discuss in the following section. Thus, the $u_{g t}$ variables are modeled as binary variables, see (16), while the $v_{g t}$ and $w_{g t}$ variables are modeled as continuous variables, see (14) and (15).

\section{E. Startup and Shutdown Binary Variables}

Generator startup and shutdown variables represent binary states. However, by carefully formulating the constraints it is possible to model them as continuous variables. By including the set of constraints (7), (20), and (21), the startup and shutdown variables take on binary solutions when the unit commitment variables are feasible - specifically, when the $u$ variables are binary. This ensures that all feasible solutions for the startup and shutdown variables are binary even if they are modeled as continuous variables. Constraints (20) and (21) are valid inequalities that are dominated by (7), (8), and (9), thereby not requiring (20) and (21) to be explicitly listed in the formulation. In other words, (7), (8), and (9) are tighter inequalities and they always satisfy (20) and (21). Therefore, our formulation ensures that all feasible solutions for the startup and shutdown variables are binary even though they are modeled as continuous variables

$$
\begin{aligned}
& v_{g t} \leq 1-u_{g, t-1}, \forall g, t \\
& w_{g t} \leq u_{g, t-1}, \forall g, t
\end{aligned}
$$

Note that with the equality constraint (7), there is no requirement to have both startup (v) and shutdown (w) variables within the formulation. Our initial formulation includes both startup and shutdown variables so that the construct of the constraints can be clearly understood. However, since only one of these sets of variables is required, it is possible to choose a formulation with either the $u$ and $v$ variables or the $u$ and $w$ variables. To formulate the problem with only $u$ and $v$ (or $u$ and $w$ ) variables, (7) can be used to replace $w(v)$ wherever $w(v)$ is present. Constraint (7) is then replaced with (7') if the $v$ variables are kept; (7) would be replaced by (7'') if the $w$ variables are kept. If the formulation is reduced to include only the $u$ and $v$ ( $u$ and $w$ ) variables, the guarantee that all feasible solutions for $v(w)$ are binary still holds; thus, $v(w)$ can still be modeled as continuous variables

$$
\begin{gathered}
v_{g t} \geq u_{g t}-u_{g, t-1}, \quad \forall g, t \\
w_{g t} \geq u_{g, t-1}-u_{g t}, \forall g, t .
\end{gathered}
$$

\section{F. Minimum Up and Down Time Constraints}

MIP formulations can be compared based on computational results and/or compared by analyzing which formulation provides a tighter representation of the minimal convex set of the MIP problem. In this section, we compare formulations based on which formulation provides a tighter polyhedral representation. For information on valid inequalities, facets, and the convex hull, refer to [23].

The minimum up and down time constraints, (8) and (9), employ the turn on/off facet defining valid inequalities [24]. Hedman et al. [25] investigated the use of valid inequalities and facets for the minimum up and down time constraints associated with the generation unit commitment problem and showed how valid inequalities for the unit commitment problem can be analyzed and improved. It also showed how constraints in alternative formulations, [26] and [27], can be improved.

With the facets in [24] and other trivial inequalities, we are able to generate the convex hull of the $u, v$ projection, i.e. the minimal convex set of the $u, v$ projection is completely represented by linear inequalities within our formulation. Many papers on unit commitment formulations do not employ facet defining valid inequalities for their minimum up and down time constraints.

A recent paper on tighter unit commitment formulations, [28], which uses only unit commitment, $u$, variables within their formulation, does not use facet defining valid inequalities for the minimum up and down time constraints. However, [29] has proven that their inequalities, which only involve $u$ variables, are facets for the $u$ projection; with their facets they are able to define the convex hull for the $u$ projection from the overall unit commitment problem. Thus, the formulation from [29] provides a tighter formulation than what is presented in [28]. We use the facets defined in [24], which are the facets that create the convex hull for the $u, v$ projection. The inequalities from [29] are not facets for the $u, v$ projection so the facets from [24] produce a tighter polyhedral representation than the inequalities from [29] when startup variables, $v$, are included.

One complicating factor with the facets listed in [29] is the number of constraints that are required. To deal with this problem, [29] proposed an efficient separation algorithm. Another option is to introduce startup and/or shutdown variables and then use the facets for the $u, v$ projection listed in [24]. Adding these binary variables is computationally conducive since they can be modeled as continuous variables and it is then possible to include the facets defined in [24], which are not problematic to generate. By adding these continuous variables, it is then possible to generate the convex hull (minimal convex set) of the $u, v$ projection; additional discussion as well as computational results showing the performance of the minimum up and down time formulation used in this paper can be found in [24]. 


\section{G. Ramp Rate Constraints}

We include intertemporal ramp rate constraints, (10) and (11), see [30], as well as ramp rate contingency constraints, (12) and (13). Constraints (10) and (11) allow for the inclusion of startup and shutdown ramp rates, which may be different than the ramp rates under continuous operation. Since startup and/or shutdown variables are included in this formulation, it is preferable to use (10) and (11) over other formulations such as (10') and (11') below, see [28], because (10) and (11) are stronger valid inequalities than (10') and (11'). It can be easily verified that the right hand side of (10) and (11) can be strictly less than the right hand side of (10') and (11') thereby providing a tighter bound on the $P_{g}$ variables. Since the startup and shutdown variables can be modeled as continuous variables as previously discussed, it is preferable to use these stronger inequalities

$$
\begin{aligned}
& P_{g 0 t}-P_{g 0, t-1} \leq R_{g}^{+} u_{g, t-1}+R_{g}^{S U}\left(1-u_{g, t-1}\right), \forall g, t \\
& P_{g 0, t-1}-P_{g 0 t} \leq R_{g}^{-} u_{g, t}+R_{g}^{S D}\left(1-u_{g, t}\right), \forall g, t
\end{aligned}
$$

Since generators receive their unit commitment schedule well in advance, they can determine the appropriate time to start ramping up (or down) their unit when they turn it on (or off) in order to meet their obligated output. However, this flexibility may be limited due to their minimum up and down time constraints or current operations. It is therefore possible for the maximum startup (or shutdown) ramp rate to be equal to the unit's $P^{\max }$. We test this model on the RTS 96 system, which does not define startup or shutdown ramp rates. We therefore assume that the maximum startup and shutdown ramp rates are equal to the unit's $P^{\max }$.

\section{H. Reserve Requirements}

Reserve requirements, such as spinning and non-spinning reserve, are typically included in unit commitment models. We do not include them within our model since we are explicitly enforcing $\mathrm{N}-1$. The primary purpose of spinning and non-spinning reserve is to ensure there is enough capacity online to survive a contingency; these constraints are used as proxies to enforce $\mathrm{N}-1$ since it is typically too computationally challenging to explicitly list every contingency.

Since our primary purpose is to examine the potential of this concept, co-optimizing the generation and network topology, in order to get more accurate results we chose to study a more complex and robust problem where $\mathrm{N}-1$ is enforced versus using proxy (reserve) constraints. There is also the issue that reserve constraints are constructed based on the network configuration, e.g. zones. There is the question as to whether reserve constraints can still achieve the necessary reliability level if the network topology changes. These proxy constraints may work for the original topology but they may not work for the new network configuration; simple theoretical examples can demonstrate this as a possibility. By explicitly enforcing $\mathrm{N}-1$ versus using reserve constraints, this is a much harder problem to solve, which we discuss in the next section.

Spinning reserve may also be used for load forecast error. The purpose of regulation reserve is for load following and forecast errors; only when regulation reserve cannot address a forecast error concern would spinning reserve be called upon to address forecast errors. Such a secondary requirement for spinning reserve would generally not compare to the stringent requirement of $\mathrm{N}-1$; enforcing $\mathrm{N}-1$ already ensures there is ample online capacity available to handle load forecast errors.

\section{MODEL MODIFICATIONS For COMPUTATIONAL TESTING}

Our problem formulation is a mixed integer linear program. The difficulties in solving these specific MIP problems arise in two independent ways. First, the treatment of multiple time periods with varying load levels make the problem size very large due to replications of variables across each load condition. Secondly, the choice of settings for the binary variables generates a combinatorial number of different problems, all of which need to be examined in order to guarantee a global solution.

While the size issues typically result in solution times that grow at a quantifiable rate, the combinatorial issues are much harder to quantify and can lead to dramatically variable solution times. However, relaxing some of the binary restrictions can lead to (easier) problems whose solutions provide lower bounds on the optimal solution of the (minimization) mixed integer program, while feasible solutions provide an upper bound on its optimal solution value. With these two values in hand, a rigorous optimality gap can be calculated that indicates the percentage difference between the provided solution and what may be possible. While reducing this optimality gap to zero is an interesting academic issue, in practice, a feasible solution with a small optimality gap is typically adequate, particularly when the input data is not known with certainty. In an operational setting, proving optimality is less important than improving the current solution; we focus here on finding the best feasible solution within a given timeframe.

\section{A. Decomposing the Optimization Problem}

Since the generation unit commitment optimal power flow problem is a very difficult problem to solve, it is a common practice within the electric industry to decompose it into subproblems. We take a similar approach: we separate the problem into two sub-problems, the main problem being the 24-hour unit commitment problem with the N-1 DCOPF formulation without transmission switching, i.e. all binary $z_{k t}$ variables are fixed to one. Once this optimization problem is solved, we move to the secondary sub-problems where we fix the unit commitment variables to their solution values from the first sub-problem and then solve for $z_{k t}$.

For the RTS 96 test case, none of the ramp rate constraints are active; thus, this problem can be decomposed into $24 \mathrm{~N}-1$ DCOPF optimal transmission switching subproblems. Solving each period separately will produce the same optimal solution as when all periods are solved within one main optimization problem since the ramp rate constraints are inactive. If there are active ramp rate constraints, the N-1 DCOPF transmission switching problem can be decomposed by first initially ignoring the ramp rate constraints and solving the 24 subproblems. Constraint violations are then applied in the next iteration. Any periods that are linked by active ramp rate constraints are combined into one subproblem and resolved. This method will ensure optimality if repeated until no further 
ramp rate constraints are active. Another method would be to remove the ramp rate constraints, solve for the optimal network topologies, fix the binary solutions, add the ramp rate constraints, and then solve for the generator dispatch variables to ensure ramp rate constraints are satisfied. This method would be faster but it will not guarantee optimality.

The process of fixing the unit commitment variables, solving for the transmission switching variables, and then fixing the transmission switching variables to solve for the new unit commitment variables is repeated. This allows us to see how the network topology can affect the unit commitment solutions and vice versa. This process can be repeated until there is no change in the solution or the solution time window is exhausted. This approach works well when a good feasible solution is needed in a limited time; the drawback is that optimality is not guaranteed.

\section{B. Software Options}

Relaxing the integrality constraint on all the binary values, or fixing their values to 0 or 1 , results in a linear program (LP). Such subproblems arise at the root and every node of a branch and bound tree that is explored by the commercial code we use, CPLEX; ensuring these solve quickly is critical. The default options of CPLEX employ the dual simplex method since this is typically effective for restarting a solution process from a parent solution in the tree. However, since our problems tend to be dual degenerate, extremely large numbers of pivots are required even when the objective function is close to its optimal value. The barrier method, which has better complexity bounds for large scale problems, performs much better at solving each subproblem and completely mitigates the advantages of the restart process, see [31]. The crossover process (that moves from an interior to a basic solution) is also ineffective for similar reasons. The use of these features can be controlled by an appropriate option file for CPLEX. Not only does this improve node solution time, but it also allows us to solve the underlying problem with fixed unit commitment and transmission switching binary variables, and thus provides a feasible starting solution for the decomposition process outlined above.

Since the unit commitment problem with fixed transmission binary variables does not decouple over time, it provides a distinct computational challenge. CPLEX incorporates a number of heuristics to determine appropriate binary values at the root node, which appear to work well in this case. It is important to ensure CPLEX is configured to look for feasible integer solutions and that cutting plane generation is turned off (since such cuts are ineffective). Just allowing processing of this nature at the root node typically takes 20 hours on a desktop workstation but provides a solution for this problem with a $1 \%$ or better optimality gap. Note that the long computation time is because we have a full N-1 DCOPF formulation along with the unit commitment problem. Most unit commitment models do not impose all N-1 contingencies along with an OPF model. Solving the unit commitment problem without including an N-1 DCOPF formulation takes only ten seconds. In order to better analyze this new concept of co-optimization of unit commitment and network topology, we chose to have a more robust optimization problem.
When solving the transmission switching problem, as indicated in [15], the techniques for closing the optimality gap, specifically improving the lower bound, are largely ineffective. To overcome this, further decomposition or parallel computation is required. We use the multithreaded option of CPLEX, coupled with options that branch based on pseudo reduced costs, emphasize finding feasible solutions, and spend significant (post processing) time improving the solution determined by the branch and bound process. While these options are effective at improving the given starting solution, several of the resulting transmission switching subproblems have optimality gaps that are larger than $4 \%$ after 20 hours of computation on 4 processor desktop machines. However, research has shown that heuristic techniques can find good feasible transmission switching solutions in reasonable timeframes, [16] and [17].

CPLEX also has an "indicator constraint" option that uses the value of a (binary) variable to indicate whether or not to include a specific constraint. The formulation presented for the optimal transmission switching problem includes a "big $\mathrm{M}$ value," which essentially removes the constraints (5a) and (5b) from the formulation whenever $z_{k t}=0$ or $N 1_{e c}=0$. We have developed a formulation that does not include the big $\mathrm{M}$ values but chooses from two sets of constraints based on the chosen value for $z_{k t}$. While this mitigates the use of the big M value in constraints (5a) and (5b), the computational results are somewhat mixed due to CPLEX having a restricted set of options to apply in this problem setting. We remain hopeful that the formulation will become more attractive as schemes to exploit indicator constraints within CPLEX mature.

\section{Test CASE: IEEE 73 Bus (RTS 96) System}

\section{A. Network Overview}

The IEEE 73-bus network, also known as the three area reliability test system 1996 (RTS 96), was created by a committee of power systems experts [32]. It is common to make modifications to the RTS 96 system. In [33] the authors removed line (11-13), shifted $480 \mathrm{MW}$ of load from bus 14,15 , 19 , and 20 to bus 13, and made other modifications as well. Buses $14,15,19$, and 20 had an original total load of $820 \mathrm{MW}$; the new total load is $340 \mathrm{MW}$. In [34] the authors decrease the thermal capacity of line (14-16) to $350 \mathrm{MW}$. For this study, we incorporated the changes mentioned above from [33] and $\left[34 .^{2}\right.$ The RTS 96 system has three identical zones; the modifications are applied to all zones.

Table I provides an overview of the RTS 96 system data. The generator cost information is an average cost based on the heat rate data presented in [35] and the fuel cost (Energy Information Administration, 2007 prices) presented in Table II. The RTS 96 system includes startup costs; shutdown costs are not defined so they are assumed to be zero. There is seasonal information for the hydro units within the RTS 96 system, all of which are assumed capable of producing at their full capacity. Table III describes the problem size for this

\footnotetext{
${ }^{2}$ Modifications in [33] included reducing the total load of several buses. To determine the new load levels at these buses, we calculated each bus' initial percentage of the original total load among these buses and allocated that bus the same percentage of the new total load.
} 
study. The RTS 96 system includes a yearly load curve. Table IV shows the hourly load levels as percentages of the base load levels. The base load levels are defined in Bus Data Table-01 from [35] for the RTS 96 system.

TABLE I

RTS 96 SYSTEM DATA

\begin{tabular}{|l|c|c|c|c|c|c|}
\hline & & \multicolumn{3}{|c|}{ Capacity (MW) } & \multicolumn{2}{c|}{ Cost (\$/MWh) } \\
\hline & No. & Total & Min & Max & Min & Max \\
\hline Transmission & 117 & 44,747 & 175 & 722 & & \\
\hline Generators & 99 & 10,215 & 12 & 400 & 0.00 & 219.95 \\
\hline Base Load & 51 & 8,547 & 53 & 745 & & \\
\hline
\end{tabular}

TABLE II

FUEL COSTS

\begin{tabular}{|c|c|c|c|}
\hline \#2 Oil & \#6 Oil & Coal & Uranium \\
\hline $15.17 \$ / \mathrm{MBtu}$ & $8.40 \$ / \mathrm{MBtu}$ & $1.78 \$ / \mathrm{MBtu}$ & $0.60 \$ / \mathrm{MBtu}$ \\
\hline
\end{tabular}

TABLE III

PROBLEM Size AND PRESOlVE STATISTICS

\begin{tabular}{|l|r|r|r|}
\hline & LP & $\begin{array}{c}\text { Unit } \\
\text { Commitment }\end{array}$ & $\begin{array}{c}\text { Trans. Switch. and } \\
\text { Unit Commitment }\end{array}$ \\
\hline Columns & 1.8 Million & 1.8 Million & 1.8 Million \\
\hline Rows & 3.9 Million & 3.9 Million & 3.9 Million \\
\hline Nonzeroes & 10.6 Million & 10.6 Million & 10.6 Million \\
\hline Binary & 0 & 2,376 & 5,184 \\
\hline Presolve & & & 1.0 Million \\
\hline Columns & 2.8 Million & 1.1 Million & 2.6 Million \\
\hline Rows & 1.0 Million & 1.3 Million & 9.2 Million \\
\hline Nonzeroes & 4.9 Million & 4.4 Million & \\
\hline
\end{tabular}

TABLE IV

HOURLY LOAD PERCENT LEVELS

\begin{tabular}{|c|c|c|c|c|c|c|c|}
\hline HR1 & HR2 & HR3 & HR4 & HR5 & HR6 & HR7 & HR8 \\
\hline 67 & 63 & 60 & 59 & 59 & 60 & 74 & 86 \\
\hline HR9 & HR10 & HR11 & HR12 & HR13 & HR14 & HR15 & HR16 \\
\hline 95 & 96 & 96 & 95 & 95 & 95 & 93 & 94 \\
\hline HR17 & HR18 & HR19 & HR20 & HR21 & HR22 & HR23 & HR24 \\
\hline 99 & 100 & 100 & 96 & 91 & 83 & 73 & 63 \\
\hline
\end{tabular}

\section{B. Results and Analysis}

As previously stated, the main optimization problem that includes the generation unit commitment with the optimal transmission switching N-1 DCOPF formulation is decomposed into two sub-problems. We first solved a 24period generation unit commitment problem with an N-1 DCOPF formulation. For this solution, all transmission elements are closed, i.e. $z_{k t}=1$ for all $k$ and $t$.

The generation unit commitment, N-1 DCOPF sub-problem produces a feasible solution with the objective value at: $\$ 3,245,997$; the optimality gap for this sub-problem is $0.33 \%$. This solution will be referred to as the "base solution" and it is in bold within Table $\mathrm{V}$; the percent savings in Table $\mathrm{V}$ are in reference to the base solution. Table VI shows the base solution's objective values for each period.

We then take this generation unit commitment solution, fix the $u_{g t}, v_{g t}$, and $w_{g t}$ variables, and then solve the $24 \mathrm{~N}-1$ DCOPF optimal transmission switching problems. By optimizing the network topology, we receive a solution of $\$ 3,165,824$, which results in a $2.5 \%$ savings as compared to the base solution when we do not perform transmission switching; the optimality gap for this sub-problem is $3 \%$.

The first generation unit commitment solution was solved when all transmission elements were closed. It is possible that the optimal unit commitment schedule may change once the network topology changes, and vice versa. Thus, we take this first transmission switching solution, fix all transmission elements to the resulting $z_{k t}$ values, and then run the unit commitment N-1 DCOPF problem again. The results show that the optimal unit commitment solution depends on the chosen network topology. With the first transmission switching solution as the set network topology, we find a new unit commitment solution with a total cost of $\$ 3,161,354$, see Table V; the sub-problem optimality gap is $0.66 \%$.

The second unit commitment schedule would be more expensive than the first one if we do not optimize the network topology. With all transmission elements in service, the total cost for the second unit commitment solution is $\$ 3,272,280$, which is higher than the first unit commitment schedule's cost of $\$ 3,245,997$ when all transmission elements are closed. Once the topology is changed, a previously more expensive unit commitment schedule is now the preferred schedule.

One key difference between the two generation unit commitment solutions is that the first solution has three peaker units that are committed for only period 10 (see Table VII). The second generation unit commitment solution never commits these units. The total startup cost for the first unit commitment solution is $\$ 56,263$ versus the second unit commitment's total startup cost of $\$ 49,157$. The unit commitments are displayed in Table VII through Table XI.

We proceeded by fixing the second unit commitment solution and resolving the N-1 DCOPF optimal transmission switching problem. We received a different solution than the first transmission switching solution, with a cost of $\$ 3,125,185$ corresponding to a percent savings of $3.7 \%$ over the base case; the optimality gap for this sub-problem is $1.9 \%$. However, this second transmission switching solution is better than the first transmission switching solution combined with either the first or the second unit commitment solution. This means that the first transmission switching solution was not optimal. The first transmission switching solution has a $3 \%$ optimality gap, indicating that sub-optimality is a possibility; the second transmission solution confirms this to be true.

By decomposing this problem, we found a solution that saves $3.7 \%$ with a value of $\$ 3,125,185$. We obtained a lower bound of $\$ 3,024,989$ from the overall unit commitment and transmission switching optimization problem. The optimality gap is therefore $3.2 \%$ for our best found feasible solution. Table V presents a summary of the solutions.

Table VI shows the individual period objective values for the base solution, i.e. the first unit commitment solution with the original network topology (no switching), the final solution, i.e. the second unit commitment solution with the second transmission switching solution, and the sub-problem optimality gaps for the final solution. Though the final solution is cheaper overall, there are some periods where the costs have increased; these hours are in bold. The higher costs are a result of choosing a different unit commitment schedule. This result further emphasizes the benefit of co-optimizing the network topology with unit commitment over multiple periods.

The final solution has an overall savings of $3.7 \%$ or over $\$ 120,000$ for this single base load day. The RTS 96 test system does not compare in size to large ISO networks. If the same savings were achieved for every day of the year, the 
savings would be over $\$ 44$ million for this medium sized IEEE test case.

TABLE V

GENERATION UC AND TRANSMISSION SWITCHING SOLUTIONS

\begin{tabular}{|l|c|c|}
\hline & No Trans. Switch & $\mathbf{1}^{\text {st }}$ Switch Sol. \\
\hline $\mathbf{1}^{\text {st }}$ Generation UC & $\mathbf{\$ 3 , 2 4 5 , 9 9 7}$ & $\$ 3,165,824$ \\
\hline Subproblem lower bound & $\$ 3,235,285$ & $\$ 3,070,581$ \\
\hline \% Savings & & $2.5 \%$ \\
\hline & $\mathbf{1}^{\text {st }}$ Switch Sol. & $\mathbf{2}^{\text {nd }}$ Switch Sol. \\
\hline $2^{\text {nd }}$ Generation UC & $\$ 3,161,354$ & $\$ 3,125,185$ \\
\hline Subproblem lower bound & $\$ 3,140,588$ & $\$ 3,065,427$ \\
\hline \% Savings & $2.6 \%$ & $3.7 \%$ \\
\hline
\end{tabular}

The most expensive units are roughly $\$ 220 / \mathrm{MWh}$. Only three of these units, units 5, 71, and 72, are ever committed in the base solution and they are only committed in period 10 , see Table VII. For the final solution, all of these units are off, see Table X. After these peaker units, the second most expensive units are roughly $\$ 100 / \mathrm{MWh}$. It may be argued that the large spread in generator costs drives the savings. As specified, there is only one hour where these most expensive units were initially dispatched. If we ignore this single period, the savings from the other 23 periods still have a total savings of $3.6 \%$. Thus, the spread in costs has an impact, but a very minor one for this test case. Table XI shows the changes in unit commitment schedules; this demonstrates how changing the network topology can alter the unit commitments.

Table XII lists the second transmission switching solution. Transmission elements that are never opened are not listed in the table. The results show that the optimal network topology may vary as network conditions vary and may be part of improved planning protocols. The differences between the two transmission switching solutions are shown in Table XIII.

Transmission switching can have a variety of impacts on market participants. As the results have shown, it can alter the optimal unit commitment solution by turning off previously committed units and committing others. The only conclusion that is possible from transmission switching is that the total social welfare will not decrease.

TABLE VI

BASE Solution Cost, FinAl SOlution Cost, AND Optimality GAP

\begin{tabular}{|l|r|r|l|r|r|r|r|r|}
\hline HR & $\begin{array}{c}\text { Final Solution } \\
\text { (Optimality Gap) }\end{array}$ & $\begin{array}{c}\text { Base UC } \\
\text { Solution }\end{array}$ & HR & $\begin{array}{c}\text { Final Solution } \\
\text { (Optimality Gap) }\end{array}$ & $\begin{array}{c}\text { Base UC } \\
\text { Solution }\end{array}$ & HR & $\begin{array}{c}\text { Final Solution } \\
\text { (Optimality Gap) }\end{array}$ & $\begin{array}{c}\text { Base UC } \\
\text { Solution }\end{array}$ \\
\hline $\mathbf{1}$ & $\mathbf{\$ 7 3 , 1 0 3 ( 0 . 1 7 \% )}$ & $\mathbf{\$ 7 0 , 8 9 9}$ & $\mathbf{9}$ & $\$ 196,392(0.82 \%)$ & $\$ 207,444$ & $\mathbf{1 7}$ & $\$ 193,197(0.14 \%)$ & $\$ 205,439$ \\
\hline $\mathbf{2}$ & $\mathbf{\$ 6 7 , 1 5 9 ( 0 . 0 1 \% )}$ & $\mathbf{\$ 6 4 , 6 0 1}$ & $\mathbf{1 0}$ & $\$ 178,792(0.66 \%)$ & $\$ 189,923$ & $\mathbf{1 8}$ & $\$ 200,193(0.04 \%)$ & $\$ 213,685$ \\
\hline $\mathbf{3}$ & $\mathbf{\$ 6 2 , 8 1 0 ( 0 p t )}$ & $\mathbf{\$ 6 0 , 3 6 8}$ & $\mathbf{1 1}$ & $\$ 185,831(0.44 \%)$ & $\$ 203,567$ & $\mathbf{1 9}$ & $\$ 200,193(0.04 \%)$ & $\$ 213,685$ \\
\hline $\mathbf{4}$ & $\mathbf{\$ 6 1 , 3 6 4 ( 0 p t )}$ & $\mathbf{\$ 5 9 , 1 3 0}$ & $\mathbf{1 2}$ & $\$ 168,172(1.94 \%)$ & $\$ 175,941$ & $\mathbf{2 0}$ & $\$ 172,192(0.35 \%)$ & $\$ 182,528$ \\
\hline $\mathbf{5}$ & $\mathbf{\$ 6 1 , 3 6 4 ( \mathbf { 0 p t } )}$ & $\mathbf{\$ 5 9 , 1 3 0}$ & $\mathbf{1 3}$ & $\$ 168,172(1.94 \%)$ & $\$ 175,941$ & $\mathbf{2 1}$ & $\$ 146,328(6.29 \%)$ & $\$ 152,407$ \\
\hline $\mathbf{6}$ & $\mathbf{\$ 6 2 , 8 3 2 ( \mathbf { 0 . 0 3 } \% )}$ & $\mathbf{\$ 6 0 , 3 6 8}$ & $\mathbf{1 4}$ & $\$ 168,172(1.94 \%)$ & $\$ 175,941$ & $\mathbf{2 2}$ & $\$ 110,333(4.05 \%)$ & $\$ 110,372$ \\
\hline $\mathbf{7}$ & $\mathbf{\$ 8 4 , 3 5 5 ( 1 . 3 6 \% )}$ & $\mathbf{\$ 8 2 , 2 2 1}$ & $\mathbf{1 5}$ & $\$ 158,505(4.61 \%)$ & $\$ 164,116$ & $\mathbf{2 3}$ & $\$ 67,519(1.36 \%)$ & $\$ 68,355$ \\
\hline $\mathbf{8}$ & $\$ 124,346(13.0 \%)$ & $\$ 129,280$ & $\mathbf{1 6}$ & $\$ 163,351(3.27 \%)$ & $\$ 169,893$ & $\mathbf{2 4}$ & $\$ 50,509(0.37 \%)$ & $\$ 50,766$ \\
\hline
\end{tabular}

TABLE VII

FirSt GENERATION UNIT COMMITMENT SOLUTION $-u_{\text {gt }}$ SOLUTION

\begin{tabular}{|l|r|r|r|r|r|r|r|r|r|r|r|r|r|r|r|r|r|r|r|r|r|r|r|r|}
\hline HR/GEN & $\mathbf{5}$ & $\mathbf{9}$ & $\mathbf{1 0}$ & $\mathbf{1 1}$ & $\mathbf{1 2}$ & $\mathbf{1 6}$ & $\mathbf{1 7}$ & $\mathbf{1 8}$ & $\mathbf{1 9}$ & $\mathbf{2 0}$ & $\mathbf{2 2}$ & $\mathbf{4 3}$ & $\mathbf{4 4}$ & $\mathbf{4 6}$ & $\mathbf{4 9}$ & $\mathbf{5 0}$ & $\mathbf{5 1}$ & $\mathbf{5 2}$ & $\mathbf{5 3}$ & $\mathbf{7 1}$ & $\mathbf{7 2}$ & $\mathbf{7 6}$ & $\mathbf{7 9}$ & $\mathbf{8 4}$ \\
\hline $\mathbf{8}$ & & & & & & 1 & 1 & 1 & 1 & 1 & & & & & 1 & 1 & & & & & & & & 1 \\
\hline $\mathbf{9}$ & & 1 & 1 & 1 & & 1 & 1 & 1 & 1 & 1 & 1 & 1 & 1 & & 1 & 1 & & 1 & & & & 1 & & 1 \\
\hline $\mathbf{1 0}$ & 1 & 1 & 1 & 1 & & 1 & 1 & 1 & 1 & 1 & 1 & 1 & 1 & & 1 & 1 & 1 & 1 & 1 & 1 & 1 & 1 & & 1 \\
\hline $\mathbf{1 1}$ & & 1 & 1 & 1 & 1 & 1 & 1 & 1 & 1 & 1 & 1 & 1 & 1 & 1 & 1 & 1 & 1 & 1 & 1 & & & 1 & 1 & 1 \\
\hline $\mathbf{1 2}$ & & 1 & 1 & 1 & 1 & & & & & & 1 & 1 & 1 & 1 & & & 1 & 1 & 1 & & & 1 & 1 & \\
\hline $\mathbf{1 3}$ & 1 & 1 & 1 & 1 & & & & & & 1 & 1 & 1 & 1 & & & 1 & 1 & 1 & & & 1 & 1 & \\
\hline $\mathbf{1 4}$ & & 1 & 1 & 1 & 1 & & & & & & 1 & 1 & 1 & 1 & & & 1 & 1 & 1 & & & 1 & 1 & \\
\hline $\mathbf{1 5}$ & 1 & 1 & 1 & 1 & & & & & & 1 & 1 & 1 & 1 & & & 1 & 1 & 1 & & & 1 & 1 & \\
\hline $\mathbf{1 6}$ & 1 & 1 & 1 & 1 & & & & & & 1 & 1 & 1 & 1 & & & 1 & 1 & 1 & & & 1 & 1 & \\
\hline $\mathbf{1 7}$ & 1 & 1 & 1 & 1 & & & & & & 1 & 1 & 1 & 1 & & & 1 & 1 & 1 & & & 1 & 1 & \\
\hline $\mathbf{1 8}$ & 1 & 1 & 1 & 1 & & & & & & 1 & 1 & 1 & 1 & & & 1 & 1 & 1 & & & 1 & 1 & \\
\hline $\mathbf{1 9}$ & 1 & 1 & 1 & 1 & & & & & & 1 & 1 & 1 & 1 & & & 1 & 1 & 1 & & & 1 & 1 & \\
\hline $\mathbf{2 0}$ & 1 & 1 & 1 & 1 & & & & & & 1 & 1 & 1 & 1 & & & & & & & & 1 & 1 & \\
\hline $\mathbf{2 1}$ & 1 & 1 & 1 & 1 & & & & & & 1 & 1 & 1 & 1 & & & & & & & & 1 & 1 & \\
\hline $\mathbf{2 2}$ & & & & & 1 & & & & & & 1 & 1 & 1 & 1 & & & & & & & & 1 & 1 & \\
\hline
\end{tabular}

TABLE VIII

FIRST GENERATION Unit COMMITMENT SOLUTION

\begin{tabular}{|l|l|}
\hline Generators Always Off: & $1,2,6,13-15,34,35,38,39,47,48,67,68,80-83,85,86$ \\
\hline Generators Always On: & $23-30,33,36,40,41,54-66,69,70,73,74,89-99$ \\
\hline Generators Off in HR 24 only & $3,4,7,8,31,32,37,88$ \\
\hline Generators Off in HR 23 and 24 only & $42,75,77$ \\
\hline Generators Off in HR 22, 23, and 24 only & $21,45,78,87$ \\
\hline
\end{tabular}


TABLE IX

\begin{tabular}{|l|r|r|r|r|r|r|r|r|r|r|r|r|r|r|}
\hline HR/GEN & $\mathbf{9}$ & $\mathbf{1 0}$ & $\mathbf{1 1}$ & $\mathbf{1 2}$ & $\mathbf{4 3}$ & $\mathbf{4 4}$ & $\mathbf{4 9}$ & $\mathbf{5 0}$ & $\mathbf{5 1}$ & $\mathbf{5 2}$ & $\mathbf{5 3}$ & $\mathbf{7 6}$ & $\mathbf{7 7}$ & $\mathbf{7 9}$ \\
\hline $\mathbf{8}$ & & & & & & & 1 & 1 & 1 & & 1 & & & \\
\hline $\mathbf{9}$ & 1 & & 1 & & 1 & 1 & 1 & 1 & 1 & 1 & 1 & 1 & 1 & \\
\hline $\mathbf{1 0}$ & 1 & 1 & 1 & & 1 & 1 & 1 & 1 & 1 & 1 & 1 & 1 & 1 & \\
\hline $\mathbf{1 1}$ & 1 & 1 & 1 & 1 & 1 & 1 & 1 & 1 & 1 & 1 & 1 & 1 & 1 & 1 \\
\hline $\mathbf{1 2}$ & 1 & 1 & 1 & 1 & 1 & 1 & & & & 1 & & 1 & 1 & 1 \\
\hline $\mathbf{1 3}$ & 1 & 1 & 1 & 1 & 1 & 1 & & & & 1 & & 1 & 1 & 1 \\
\hline $\mathbf{1 4}$ & 1 & 1 & 1 & 1 & 1 & 1 & & & & 1 & & 1 & 1 & 1 \\
\hline $\mathbf{1 5}$ & 1 & 1 & 1 & 1 & 1 & 1 & & & & 1 & & 1 & 1 & 1 \\
\hline $\mathbf{1 6}$ & 1 & 1 & 1 & 1 & 1 & 1 & & & & 1 & & 1 & 1 & 1 \\
\hline $\mathbf{1 7}$ & 1 & 1 & 1 & 1 & 1 & 1 & & & & 1 & & 1 & 1 & 1 \\
\hline $\mathbf{1 8}$ & 1 & 1 & 1 & 1 & 1 & 1 & & & & 1 & & 1 & 1 & 1 \\
\hline $\mathbf{1 9}$ & 1 & 1 & 1 & 1 & 1 & 1 & & & & 1 & & 1 & 1 & 1 \\
\hline $\mathbf{2 0}$ & 1 & & & 1 & 1 & 1 & & & & & & 1 & 1 & 1 \\
\hline $\mathbf{2 1}$ & & & & 1 & 1 & 1 & & & & & & 1 & 1 & 1 \\
\hline $\mathbf{2 2}$ & & & & 1 & 1 & 1 & & & & & & 1 & 1 & 1 \\
\hline
\end{tabular}

TABLE X

SECOND GENERATION UNIT COMMITMENT SOLUTION

\begin{tabular}{|l|l|}
\hline Generators Always Off: & $1,2,5,6,13-20,34,35,38,39,47,48,67,68,71,72,80-86$ \\
\hline Generators Always On: & $23-30,33,36,40,41,54-66,69,70,73,74,89-99$ \\
\hline Generators Off in HR 24 only & $3,4,7,8,31,32,37,88$ \\
\hline Generators Off in HR 23 and 24 only & $22,42,46,75$ \\
\hline Generators Off in HR 22, 23, and 24 only & $21,45,78,87$ \\
\hline
\end{tabular}

TABLE XI

GENERATION Unit COMMITMENT CHANGES BETwEen SOLUTION 1 AND 2

\begin{tabular}{|c|c|c|c|c|c|c|c|c|}
\hline \multicolumn{6}{|c|}{ Units dispatched in fewer periods } & \multicolumn{3}{|c|}{ Units dispatched in more periods } \\
\hline & \multicolumn{2}{|c|}{ Periods } & \multirow[b]{2}{*}{ Generator } & \multicolumn{2}{|c|}{ Periods } & \multirow{2}{*}{ Generator } & \multicolumn{2}{|c|}{ Periods } \\
\hline Generator & UC1 & UC2 & & UC1 & UC2 & & UC1 & UC2 \\
\hline $5,71,72$ & 10 & None & $16-20,84$ & $8-11$ & None & 22 & $9-22$ & $1-22$ \\
\hline 9 & $9-21$ & $9-20$ & 51,53 & $10-19$ & $8-11$ & 46 & $11-22$ & $1-22$ \\
\hline 10 & $9-21$ & $10-19$ & 77 & $1-22$ & $9-22$ & & & \\
\hline 11 & $9-21$ & $9-19$ & & & & & & \\
\hline
\end{tabular}

TABLE XII

SECOND Transmission Switching SOlution (LinEs Not Listed ARE Always Closed) $-z_{k t}$ Solution

\begin{tabular}{|l|r|r|r|r|r|r|r|r|r|r|r|r|r|r|}
\hline HR/Line & $\mathbf{1 5}$ & $\mathbf{2 0}$ & $\mathbf{2 3}$ & $\mathbf{4 1}$ & $\mathbf{5 4}$ & $\mathbf{6 3}$ & $\mathbf{6 4}$ & $\mathbf{6 9}$ & $\mathbf{7 2}$ & $\mathbf{7 3}$ & $\mathbf{7 5}$ & $\mathbf{9 1}$ & $\mathbf{1 0 8}$ & $\mathbf{1 0 9}$ \\
\hline $\mathbf{1}$ & & 1 & & & 1 & 1 & 1 & 1 & 1 & 1 & 1 & & 1 & 1 \\
\hline $\mathbf{2}$ & 1 & 1 & & 1 & 1 & 1 & 1 & & 1 & 1 & 1 & & 1 & 1 \\
\hline $\mathbf{4}$ & 1 & 1 & & 1 & 1 & 1 & 1 & & 1 & 1 & 1 & & 1 & 1 \\
\hline $\mathbf{5}$ & 1 & 1 & & 1 & 1 & 1 & 1 & & 1 & 1 & 1 & 1 & 1 & 1 \\
\hline $\mathbf{6}$ & 1 & 1 & & 1 & 1 & 1 & 1 & & 1 & 1 & 1 & 1 & 1 & 1 \\
\hline $\mathbf{7}$ & & 1 & & & & 1 & 1 & & 1 & 1 & 1 & & 1 & \\
\hline $\mathbf{8}$ & 1 & 1 & 1 & 1 & 1 & 1 & 1 & & 1 & 1 & 1 & & & \\
\hline $\mathbf{9}$ & & 1 & 1 & 1 & 1 & & & 1 & 1 & 1 & & & & \\
\hline $\mathbf{1 0}$ & & 1 & 1 & 1 & 1 & 1 & 1 & & 1 & & & & & \\
\hline $\mathbf{1 1}$ & & 1 & & 1 & & 1 & 1 & & 1 & 1 & 1 & & 1 & 1 \\
\hline $\mathbf{1 2}$ & & 1 & 1 & 1 & 1 & & & 1 & 1 & 1 & & & & \\
\hline $\mathbf{1 3}$ & & 1 & 1 & 1 & 1 & & & 1 & 1 & 1 & & & & \\
\hline $\mathbf{1 4}$ & & 1 & 1 & 1 & 1 & & & 1 & 1 & 1 & & & & \\
\hline $\mathbf{1 5}$ & & 1 & 1 & 1 & 1 & 1 & 1 & & 1 & 1 & & & & \\
\hline $\mathbf{1 6}$ & & 1 & 1 & 1 & 1 & 1 & 1 & & 1 & 1 & & & & \\
\hline $\mathbf{1 7}$ & & 1 & 1 & 1 & 1 & & & 1 & 1 & 1 & & & 1 & 1 \\
\hline $\mathbf{1 8}$ & & 1 & 1 & 1 & 1 & 1 & 1 & 1 & & 1 & 1 & & 1 & 1 \\
\hline $\mathbf{1 9}$ & & 1 & 1 & 1 & 1 & 1 & 1 & 1 & & 1 & 1 & & 1 & 1 \\
\hline $\mathbf{2 0}$ & & 1 & 1 & 1 & 1 & 1 & 1 & & 1 & & & & 1 & \\
\hline $\mathbf{2 1}$ & & 1 & 1 & 1 & 1 & 1 & 1 & & 1 & 1 & 1 & & & \\
\hline $\mathbf{2 2}$ & 1 & 1 & 1 & & 1 & 1 & 1 & & 1 & 1 & 1 & 1 & 1 & 1 \\
\hline $\mathbf{2 3}$ & 1 & 1 & & 1 & & 1 & 1 & 1 & 1 & 1 & 1 & 1 & 1 & 1 \\
\hline $\mathbf{2 4}$ & 1 & & 1 & & 1 & 1 & 1 & & 1 & 1 & 1 & & & \\
\hline
\end{tabular}


TABLE XIII

Changes Between The First and SeCond Transmission Switching Solutions

\begin{tabular}{|c|c|c|c|c|c|c|c|c|c|c|c|c|c|c|c|c|c|c|c|c|c|c|c|}
\hline HR/Line & 1 & 15 & 16 & 18 & 20 & 23 & 31 & 37 & 41 & 54 & 63 & 64 & 69 & 72 & 73 & 75 & 91 & 93 & 100 & 106 & 108 & 109 & 112 \\
\hline 1 & & & & & & & & & & & & & + & & & & & & & & & & + \\
\hline 2 & & & & & & & & & & & & & & & & & $*$ & + & & & & & \\
\hline 3 & & & & & & $*$ & & & & & & & & & & & $*$ & & & & & + & \\
\hline 4 & & & + & & & & & & & & & & $*$ & & & & & & & & & & \\
\hline 5 & & & & + & & & & & & & & & & & & & & & & & & & \\
\hline 6 & + & & & & & & & & & & & & & & & & 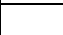 & & & & & & \\
\hline 7 & & $*$ & & & & & & & & & & & $*$ & & & & & & & & & & \\
\hline 8 & & & & & & & & & & & & & $*$ & & & & & & & & $*$ & $*$ & \\
\hline 9 & & & & & & & & & & & $*$ & $*$ & + & & & $*$ & & & & & $*$ & $*$ & \\
\hline 10 & & $*$ & & & & & & & & & & & & & $*$ & $*$ & & & & & $*$ & $*$ & \\
\hline 11 & & & & & & $*$ & & & & & & & & & & & & & & + & & 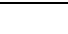 & \\
\hline 12 & & & & & & + & + & & & + & $*$ & $*$ & + & & & & & & & & $*$ & & \\
\hline 13 & & & & & & & & & & & $*$ & $*$ & & & & $*$ & & & & . & $*$ & $*$ & \\
\hline 14 & & & & & & & & & & & $*$ & $*$ & + & & + & & & & & + & $*$ & $*$ & \\
\hline 15 & & & & & & & & & & & & & $*$ & & & $*$ & & & & & $*$ & $*$ & \\
\hline 16 & & $*$ & & & & & & & & & & & & & & & & & & + & $*$ & $*$ & + \\
\hline 17 & & & & & & & & & & & $*$ & $*$ & & & & $*$ & & & + & & & & \\
\hline 18 & & $*$ & & & & & + & & & & & & + & $*$ & & & & & & + & & & \\
\hline 19 & & $*$ & & & & & + & & & & & & + & $*$ & & & & & & + & & & \\
\hline 20 & & & & & & & & + & & & & & & & & $*$ & & & & & + & & \\
\hline 21 & & & & & & & & & & & & & $*$ & & & & $*$ & & & & $*$ & $*$ & \\
\hline 22 & & & & & & & & & $*$ & & & & & & & & & & & & & & \\
\hline 23 & & & & & & & & & & $*$ & & & & & & & & & & & & & \\
\hline 24 & & & & & $*$ & & & & & & & & & & & & $*$ & & & & $*$ & $*$ & \\
\hline
\end{tabular}

\section{DISCUSSION AND FUTURE WORK}

Transmission switching has been shown to provide savings when solving the network with a generation unit commitment N-1 DCOPF problem. Future research could investigate dynamic load patterns to research the effects of transmission switching in an $\mathrm{AC}$ formulation since lines affect reactive power profiles differently under different loading patterns. There is also the need to research the impacts from transmission switching regarding real time operations including voltage profiles, reactive power, transient stability, etc. This analysis is necessary at varying load levels as well since the capacitive component of a transmission element is predominant during low load levels whereas the reactive component is predominant at higher load levels. Research into whether transmission switching would be beneficial for large scale, practical networks and whether solutions can be obtained within a reasonable timeframe is needed as well. Further, research could look into geographic decomposition.

The RTS 96 system is a standard IEEE test case. It is large enough to provide meaningful results but it is considerably smaller than the ISO markets. Solving this problem took large, specialized computer networks and even with this equipment solving the problems took well over 20 hours at each stage. The transmission switching problem with a fixed generator unit commitment could be run overnight to find a better transmission dispatch. For this method to be practically implemented, research on how to solve this problem faster for larger networks is needed.

The large computational times do not suggest that this approach of co-optimizing transmission topology with generation unit commitment and dispatch is not possible. As previously mentioned, the main difficulty is that we are combining unit commitment with a full N-1 DCOPF formulation, something that is not done in a practical setting.
In operational networks, the operator may use a security constrained unit commitment (SCUC) with multiple stages that may include network constraints and chosen contingency constraints; however, the unit commitment stage still does not include a full N-1 OPF formulation. We wish to study a more robust formulation in order to obtain stronger, more accurate conclusions. Future research should investigate how to best decompose this large optimization problem in the same way unit commitment is handled within a practical setting today. Furthermore, future research should consider whether reserve constraints are appropriate proxy constraints for $\mathrm{N}-1$ within transmission switching models.

As more is learned about the network and transmission switching, operators will know which transmission elements are candidates for switching so that there is no need to represent every transmission element within the network with a binary decision variable reflecting whether the element will be closed or not. Rather, the operator may be able to focus on a subset of transmission elements that are key candidates for switching, which will greatly reduce the number of binary variables in the optimization problem and, thus, reduce the computational complexity of the problem.

While the market surplus increases in the solution process, there is no assurance that the load ends up paying less or the generators receive more profit under traditional settlement rules (see [16] and [17]). Revenue adequacy for FTRs is maintained for the static DC network [36], but not guaranteed if the network topology changes [37]. Revenue inadequacy as a result of transmission switching is possible even though the market surplus increases. Even if there is revenue inadequacy, since the total surplus is guaranteed not to decrease with transmission switching, there is the possibility for Pareto improvements for all market participants. This raises the question about the settlement rules if revenue inadequacy occurs. 


\section{CONCLUSIONS}

As computing power and optimization techniques improve, the multi-trillion dollar electric industry looks for ways to cut costs by taking advantage of these improvements. Viewing transmission elements as committable assets in an optimization framework is relatively new as such analysis was not possible in the past due to the complexity this added to an already challenging problem. As computing power increases and software improves, potential annual savings may be in the tens of billion dollars by improving the dispatch and making better investments.

There are concerns with whether transmission switching will be a detriment to reliability. We have demonstrated that a network can satisfy N-1 standards while cutting costs by cooptimizing the network topology and the dispatch. We have also demonstrated that changing the topology of the network can change the optimal unit commitment schedule and it may reduce startup costs. This demonstrates that changing the topology can replace the need to startup a generator. Our work thus far has shown substantial savings from transmission switching, with this study showing a $\$ 120,000$ savings for one day. These findings suggest that further research on transmission switching is justified for larger networks and with more granular modeling, such as an ACOPF.

\section{REFERENCES}

[1] A. Ott, VP, PJM, Norristown, PA, private communication, July 2008.

[2] S. Nauman, VP, Exelon, Chicago, IL, private communication, July 2008.

[3] Northeast Power Coordinating Council, Guidelines for inter-area voltage control. New York, NY: NPCC Operating Procedure Coordinating Committee and NPCC System Design Coordinating Committee, 1997.

[4] ISO-NE, ISO New England Operating Procedure no. 19: Transmission Operations, pp. 7-8, Apr. 13, 2007.

[5] G. Granelli, M. Montagna, F. Zanellini, P. Bresesti, R. Vailati, and M. Innorta, "Optimal network reconfiguration for congestion management by deterministic and genetic algorithms," Electr. Power Syst. Res., vol. 76, no. 6-7, pp. 549-556, Apr. 2006.

[6] W. Shao and V. Vittal, "BIP-based OPF for line and bus-bar switching to relieve overloads and voltage violations," in Proc. 2006 IEEE Power System Conf. and Expo., Atlanta, GA, October 29 - November 1, 2006.

[7] A. G. Bakirtzis and A. P. (Sakis) Meliopoulos, "Incorporation of switching operations in power system corrective control computations," IEEE Trans. Power Syst., vol. 2, no. 3, pp. 669-675, Aug. 1987.

[8] G. Schnyder and H. Glavitsch, "Security enhancement using an optimal switching power flow," IEEE Trans. Power Syst., vol. 5, no. 2, pp. 674681, May 1990.

[9] R. Bacher and H. Glavitsch, "Loss reduction by network switching," IEEE Trans. Power Syst., vol. 3, no. 2, pp. 447-454, May 1988.

[10] G. Schnyder and H. Glavitsch, "Integrated security control using an optimal power flow and switching concepts," IEEE Trans. Power Syst., vol. 3, no. 2, pp. 782-790, May 1988.

[11] R. Bacher and H. Glavitsch, "Network topology optimization with security constraints," IEEE Trans. Power Syst., vol. 1, no. 4, pp. 103111, Nov. 1986.

[12] W. Shao and V. Vittal, "Corrective switching algorithm for relieving overloads and voltage violations," IEEE Trans. Power Syst., vol. 20, no. 4, pp. 1877-1885, Nov. 2005.

[13] B. G. Gorenstin, L. A. Terry, M. V. F. Pereira, and L. M. V. G. Pinto, "Integrated network topology optimization and generation rescheduling for power system security applications," in IASTED INTL. SYMP.: High Tech. in the Power Industry, pp. 110-114, Bozeman, MT, Aug. 1986.

[14] R. P. O'Neill, R. Baldick, U. Helman, M. H. Rothkopf, and W. Stewart, "Dispatchable transmission in RTO markets," IEEE Trans. Power Syst., vol. 20, no. 1, pp. 171-179, Feb. 2005.

[15] E. B. Fisher, R. P. O'Neill, and M. C. Ferris, "Optimal transmission switching," IEEE Trans Power Syst., vol. 23, no. 3, pp. 1346-1355, Aug. 2008.
[16] K. W. Hedman, R. P. O’Neill, E. B. Fisher, and S. S. Oren, "Optimal transmission switching - sensitivity analysis and extensions," IEEE Trans. Power Syst., vol. 23, no. 3, pp. 1469-1479, Aug. 2008.

[17] K. W. Hedman, R. P. O’Neill, E. B. Fisher, and S. S. Oren, "Optimal transmission switching with contingency analysis," IEEE Trans. Power Syst., vol. 24, no. 3, Aug. 2009.

[18] S. P. Vajjhala and P. S. Fischbeck, "Quantifying siting difficulty: A case study of US transmission line siting," Energy Policy, vol. 35, no. 1, pp. 650-671, 2007.

[19] Entrepreneur, "PJM scheduling software saves customers millions," Aug. 2004. [Online]. Available: http://www.entrepreneur.com/tradejournals/article/119368242.html.

[20] R. P. O'Neill, "It's getting better all the time (with mixed integer programming)," in HEPG Forty-Ninth Plenary Session, Dec., 2007. [Online]. Available: http://www.hks.harvard.edu/hepg/ONeill_Richard.pdf.

[21] S. Binato, M. V. F. Pereira, and S. Granville, "A new Benders decomposition approach to solve power transmission network design problems," IEEE Trans. Power Syst., vol. 16, no. 2, pp. 235-240, May 2001.

[22] L. L. Garver, "Power generation scheduling by integer programming development of theory," AIEE Trans., vol. 81, no. 3, pp. 730-734, Apr. 1962.

[23] G. L. Nemhauser and L. A. Wolsey, Integer and Combinatorial Optimization. New York: Wiley-Interscience, 1999.

[24] D. Rajan and S. Takriti, "Minimum up/down polytopes of the unit commitment problem with start-up costs," IBM Research Report, June 2005. [Online]. Available: http://www.research.ibm.com/people/d/dpkrjn/DeepakTR.pdf.

[25] K. W. Hedman, R. P. O'Neill, and S. S. Oren, "Analyzing valid inequalities of the generation unit commitment problem," in Proc. 2009 IEEE Power System Conf. and Expo., Seattle, WA, March 2009.

[26] T. Li and M. Shahidehpour, "Price-based unit commitment: a case of Lagrangian relaxation versus mixed integer programming," IEEE Trans. Power Syst., vol. 20, no. 4, pp. 2015-2025, Nov. 2005.

[27] G. W. Chang, Y. D. Tsai, C. Y. Lai, and J. S. Chung, "A practical mixed integer linear programming based approach for unit commitment," IEEE PES General Meeting 2004, vol. 1, pp. 221-225, June 2004.

[28] A. Frangioni, C. Gentile, and F. Lacalandra, "Tighter approximated MILP formulations for unit commitment problems," IEEE Trans. Power Syst., vol. 24, no. 1, pp. 105-113, Feb. 2009.

[29] J. Lee, J. Leung, and F. Margot, "Min-up/min-down polytopes," Discrete Optimization, vol. 1, no. 1, pp. 77-85, June 2004.

[30] J. M. Arroyo and A. J. Conejo, "Optimal response of a thermal unit to an electricity spot market," IEEE Trans. Power Syst., vol. 15, no. 3, pp. 1098-1104, Aug. 2000.

[31] S. J. Wright, Primal-Dual Interior-Point Methods. Philadelphia, PA: SIAM, 1997, ch. 11.

[32] C. Grigg, P. Wong, et al., "The IEEE reliability test system-1996. A report prepared by the reliability test system task force of the application of probability methods subcommittee," IEEE Trans. Power Syst., vol. 14, no 3, pp. 1010-1020, Aug. 1999.

[33] J. McCalley, S. Asgarpoor, et al., "Probabilistic security assessment for power system operations," IEEE PES General Meeting, 2004. vol. 1, pp 212-220, June 2004.

[34] A. L. Motto, F. D. Galiana, A. J. Conejo, and J. M. Arroyo, "Networkconstrained multiperiod auction for a pool-based electricity market," IEEE Trans. Power Syst., vol. 17, no. 3, pp 646-653, Aug. 2002.

[35] Power System Test Case Archive, Univ. Washington, Dept Elect. Eng., 2007. [Online]. Available: https://www.ee.washington.edu/research/pstca/index.html.

[36] R. P. O'Neill, U. Helman, B. F. Hobbs, W. R. Stewart, Jr., and M. H. Rothkopf, "A joint energy and transmission rights auction for RTO markets," in Proc. IEE $5^{\text {th }}$ International Conf. on Power System Management and Control, London, April 2002.

[37] O. Alsac, J. M. Bright, S. Brignone, M. Prais, C. Silva, B. Scott, and N. Vempati, "The rights to fight price volatility." IEEE Power and Energy Magazine, vol. 2, no. 4, pp. 47-57, 2004.

Kory W. Hedman (S'05) received the B.S. degree in electrical engineering and the B.S. degree in economics from the University of Washington, Seattle, in 2004 and the M.S. degree in economics and the M.S. degree in electrical engineering (thesis option) from Iowa State University, Ames, in 2006 and 2007, respectively. He is pursuing the Ph.D. degree at the University of 
California, Berkeley in the Department of Industrial Engineering and Operations Research; he received the M.S. degree in IEOR from the same department in 2007.

He previously worked for the California ISO (CAISO), Folsom, CA, on transmission planning and he has worked with the Federal Energy Regulatory Commission (FERC), Washington, DC, on transmission switching.

Michael C. Ferris is a Professor of computer sciences and industrial and systems engineering at the University of Wisconsin-Madison. His research interests are in computational algorithms and modeling, coupled with applications in economics, structural, and mechanical engineering and medicine. His work emphasizes practical solution of large-scale optimization and complementarity problems.

Professor Ferris is a member of the Mathematical Programming Society, SIAM, and a fellow of INFORMS.

Richard P. O'Neill received the B.S. degree in chemical engineering and the $\mathrm{Ph} . \mathrm{D}$. degree in operations research from the University of Maryland at College Park.

Currently, he is the Chief Economic Advisor in the Federal Energy Regulatory Commission (FERC), Washington, DC. He was previously on the faculty of the Department of Computer Science, Louisiana State University, and the Business School at the University of Maryland at College Park.

Emily Bartholomew Fisher (S'05) received the B.S. degree in electrical engineering from Brown University, Providence, RI. She is pursuing the Ph.D. degree in energy economics and systems at Johns Hopkins University, Baltimore, MD.

She is also a part-time researcher with the Washington DC offices of Lawrence Berkeley National Laboratory. She was formerly an engineer in the Office of Energy Market Regulation at the Federal Energy Regulatory Commission (FERC), Washington, DC.

Shmuel S. Oren (M'72-SM'99-F'02) received the B.Sc. and M.Sc. degrees in mechanical engineering and in materials engineering from the Technion Haifa, Israel, and the MS. and Ph.D. degrees in engineering economic systems from Stanford University, Stanford, CA, in 1972.

$\mathrm{He}$ is a Professor of IEOR at the University of California at Berkeley. He is the Berkeley site director of the Power System Engineering Research Center (PSERC). He has published numerous articles on aspects of electricity market design and has been a consultant to various private and government organizations including the Brazilian regulatory commission, the Alberta Energy Utility Board, the Public Utilities Commission, the Polish system operator, and the Public Utilities Commission of Texas, where he is currently a Senior Advisor to the Market Oversight Division.

Dr. Oren is a Fellow of INFORMS. 